\title{
Transurethral use of nephroscope in the management of bladder stone
}

\author{
G S Sigdel, ${ }^{1}$ A Agarwal, ${ }^{2}$ N Lamichhane, ${ }^{3}$ W K Belokar ${ }^{4}$ \\ 1,2,3 Residents Mch Urology, ${ }^{4}$ Prof. and HOD, Department of Urology, College of Medical Sciences, Teaching \\ Hospital, Bharatpur, Chitwan, Nepal
}

\section{Abstract \\ Objective}

Endoscopic management of bladder stone is a well established procedure. The objective of this study was to evaluate the usefulness of transurethral use of nephroscope in the management of bladder stones.

\section{Materials and methods}

We retrospectively analyzed the medical records of patients who had undergone treatment of bladder stones through transurethral use of nephroscope in our hospital. Various demographic, stone related, intraoperative and postoperative parameters were studied.

\section{Results}

The mean stone size was $18.1 \pm 6.8$ millimetres. Mean operative time and duration of hospital stay were 42.2 minutes and 2.4 days respectively. Intraoperative and postoperative complications occurred in 8 and 16 percent respectively, which were minor and managed without further interventions. Stone free rate was 100 percent.

\section{Conclusion}

Bladder stones in adults can be effectively managed with the transurethral use of nephroscope without significant complications.

\section{Key words: Bladder stone, transurethral use of nephroscope.}

\section{Introduction}

Surgical treatment of bladder calculi has evolved over years from open cystolithotomy to endourological procedures including blind or optical cystolitholapaxy, cystoscopic or percutaneous cystolithotripsy and extracorporeal shock wave lithotripsy (ESWL). The benefit of endourological management includes less pain, shorter hospital stay and it can be combined with

Correspondence: Dr. Ghanshyam Sigdel E-mail: sigdelgs@yahoo.com corrective procedure for bladder outlet obstruction. ${ }^{1}$ Transurethral removal of bladder stone using nephroscope is fast and effective method compared to endoscopic treatment via cystoscope. ${ }^{2}$ In this article we aimed to review our experience of bladder stone management with transurethral use of nephroscope.

\section{Materials and methods}

A retrospective review of patients from Jan 2011 to Jan 2012 who underwent transurethral removal of 
bladder stones with the use of nephroscope was done. All patients were evaluated clinically, and were investigated with routine and microscopic urinalysis, urine culture, serum creatinine, ultrasonography of the abdomen and pelvis and x-ray kidney, ureter and bladder (KUB). The diagnosis of bladder stone was based on ultrasonography and x-ray KUB. Preliminary cystoscopy was done to define the stone related parameters like number, size and type, check for other bladder and urethral pathology. A 26 Fr. Nephroscope was introduced per urethra with its sheath. Pneumatic lithotrpsy was done using Nidhilith ${ }^{\mathrm{TM}}$ lithotrite to fragment the stone. Smaller fragments were allowed to pass spontaneously with the jet of irrigation, while larger fragments were removed manually using nephroscope forceps. Patients requiring additional endoscopic procedures like optical internal urethrotomy (OIU), urethral dilatation, transurethral resection of the prostate (TURP) and ureterorenoscopy (URS) were done in the same setting. An indwelling Foley catheter was left at the end of the procedure. All patients received 1 gram of injection ceftriaxone as prophylactic antibiotic before getting anesthesia. X-ray KUB was done before discharging the patient to assure them of total clearance of the stone. Patients' medical records were analyzed in terms of various demographic, stone related, intraoperative and postoperative parameters.

\section{Results}

A total of 25 patients were treated with the per urethral nephroscopic pneumatic lithotripsy for bladder stone from April 2010 to January 2012. None of the patients had previous history of treatment for bladder stones. There was male predominance with male to female ratio of 24: 1 (Table 1). Majority of the patients had dysuria and acute retention of urine as the presenting

symptoms. Twenty three patients had a single bladder stone, two had two stones. Three patients had associated upper urinary tract calculus disease. Ten patients (40\%) had a component of bladder outflow obstruction comprising of benign prostatic hyperplasia $(\mathrm{BPH})$, urethral stricture and neurogenic bladder (Table1). All patients were stone free in the immediate postoperative period (Table2). Two patients had minor intraoperative complications of bladder mucosal injury. Postoperative complications occurred in four patients comprised of gross hematuria in two, and urinary tract infections in two. Foley catheter was removed on the first postoperative day in patients in whom only lithotripsy was done, except in two who had gross hematuria on the first postoperative day. Patients who had undergone concomitant TURP had their catheter removed on the fifth postoperative day. In patients following concomittent OIU/ urethral dilatation catheter was removed on the seventh postoperative day.

\section{Table 1: Demographic and stone related data}

\begin{tabular}{lc}
\hline Variable(s) & Values \\
\hline Total number of patients & 25 \\
Gender (Male/female) & $24 / 1$ \\
Age in years* & $48 \pm 19.90$ (19-85) \\
Clinical presentations & 22 \\
Dysuria & 17 \\
Acute urinary retention & 14 \\
Increased frequency & 7 \\
Gross hematuria & 7 \\
Recurrent UTI & $30 \pm 44(1-150)$ \\
Duration of symptoms, Days* & $18.1 \pm 6.8(10-31)$ \\
Stone size in millimetres** & \\
Other urinary tract pathology & 7 \\
BPH & \\
\hline
\end{tabular}


Urethral stricture

Neurogenic bladder

Right nephrolithiasis

1

Left distal ureteric calculi

2

Co-morbidities

Diabetes

Hemophilia

1

Table 2: Operative and postoperative data

\begin{tabular}{lc}
\hline Variable(s) & Values \\
\hline Operative time (minutes)* & $42.2 \pm 12.4(20-60)$ \\
Stone free rate & $25(100 \%)$ \\
Hospital stay( days)* & $2.4 \pm 1.2(1-5)$ \\
Retreatment rate & 0 \\
Intraoperative complications & $2(8 \%)$ \\
Postoperative complications & $4(16 \%)$ \\
Postoperative uretheral & \\
catheter(days)* & \\
Additional procedures & $2.5 \pm 2.1(1-7)$ \\
TURP & \\
OIU/Urethral dilatation & 6 \\
URS & 2 \\
\hline
\end{tabular}

* Mean \pm SD (range)

\section{Discussion}

Urinary bladder stone accounts for nearly $5 \%$ of urinary system Calculi. ${ }^{3}$ Bladder calculi are classified as migrant or secondary calculi. ${ }^{4}$ Migrant bladder stones are formed in the upper tracts, pass into the bladder, and are retained there. The primary etiology of the calculus is related to the metabolic factors associated with renal calculi formation. Secondary

bladder calculi are formed in association with bladder outlet obstruction, recurrent urinary tract infections, neurogenic bladder dysfunction or foreign bodies in the bladder. ${ }^{4}$

Various treatment options are available for the removal of bladder stones. Open surgery remains the main treatment of bladder calculus in children and large stone burden in adult. ${ }^{5}$ It is associated with more postoperative pain, prolonged hospital stay and wound scar. Extracorporeal shock wave lithotripsy (ESWL) is non invasive, with low morbidity, without need for anesthesia, and can be done on outpatient basis; however it is less effective, may require several sessions as well as other additional procedures to clear stone fragments. ESWL cannot be done in patients with bladder outlet obstruction. ${ }^{6}$ Percutaneous suprapubic cystolithotripsy is a minimally invasive, safe and effective procedure especially for the management of larger bladder stones. ${ }^{7}$ This procedure is very useful in children by means of which the demerits of urethral manipulations and open surgery both are eliminated. Percutaneous stone removal might be the procedure of choice for managing calculi in the augmented bladder. ${ }^{8}$ All endoscopic procedures aim to achieve complete stone-free state in shortest possible time, with short hospital stay and minimal complications. Transurethral endoscopic removal is the treatment of choice for bladder calculus in adult population, not only due to scar less procedure but also due to its advantage of doing corrective surgery for associated bladder outlet pathology like benign hyperplasia of the prostate. Transurethral bladder stone management is done either by cystoscope or nephroscope. Nephroscope has distinct advantage over the cystoscope as it has a wider 
lumen, which facilitates easy removal of the stone fragments. ${ }^{9}$ Also cystoscopic fragmentation requires longer operating time and there is a decrease in vision quality, which parallels the degree of stone fragmentation. Pneumatic lithotripsy is readily available, cheaper and safe procedure compared to other forms of intracorporeal lithotripsy.

The operative time was significantly less in patients whose bladder stones were treated with transurethral nephroscope compared to others treated with transurethral cystocope or percutaneous stone removal. ${ }^{9}$ In our study the operative time (mean $\pm \mathrm{SD}$ ) was $42.2 \pm 12.4$ minutes which was comparable with 48.2 +/- 13.2 minutes, reported by Ener $\mathrm{K}$ et al. ${ }^{2}$ The hospital stay was significantly shorter after endourological procedures compared to open surgery (2.6 vs 4.8 days, $\mathrm{p}<0.05$ ). ${ }^{10}$ In our study hospital stay was $2.4 \pm 1.2$ days. Both the intraoperative and postoperative complications were minor and managed without additional interventions or morbidities. Stone free rate of 100 percent was achieved in the immediate postoperative period, which is very important from patient satisfaction point of view. Though our study is not a comparative study we found transurethral nephroscopic removal of bladder stones was quick and easy procedure without major complications. Operative time, postoperative complications and morbidities were comparable to other studies. ${ }^{9,11}$

\section{Conclusion}

Bladder stones in adult can be effectively managed with the transurethral use of nephroscope with minimal complication rate. Further prospective studies are recommended to compare the superiority of this technique over other modalities of bladder stone management.

\section{References}

1. Asci R, Aybek Z, Sarikaya S. et al. The management of vesical calculi with optical mechanical cystolithotripsy and transurethral prostatectomy is it safe and effective? BJU Inter. 1999; 84:332-6.

2. Ener K, Agras K, Aldemir M, et al. The randomized comparison of two different endoscopic techniques in the management of large bladder stones: transurethral use of nephroscope or cystoscope? J Endourol. 2009; 23:1151-5.

3. Schwartz BF, Stoller ML. The Vesical calculus. Urol Clin North Am. 2000; 27: 333-46.

4. Wein AJ, Kavoussi LR, Novick AC, et al. Campbell-Walsh Urology $9^{\text {th }}$ edition. Philadelphia , Saunders Elsevier, 2007: Page 2663.

5. Papatsoris AG, Varkarakis I, Dellis A, et al. Bladder lithiasis: from open surgery to lithotripsy. Urol Res. 2006; 34 :163-7.

6. García C JV, González EC, Cabrera PJ, et al.Bladder calculi. Is extracorporeal shock wave lithotripsy the first choice treatment? Arch Esp Urol. 2003;56 :1111-6.

7. Wollin TA, Singal RK, Whelan T, et al. Percutaneous suprapubic cystolithotripsy for treatment of large bladder calculi. J Endourol. 1999;13: 739-44.

8. Cain MP, Casale AJ, Kaefer $\mathrm{M}$ et al. Percutaneous cystolithotomy in the pediatric augmented bladder. J Urol. 2002 ; 168 :1881-2. 
9. Singh KJ, Kaur J. Comparison of three different endoscopic techniques in management of bladder calculi. Indian J Urol. 2011 ; 27:10-3.

10. Al-Marhoon MS, Sarhan OM, Awad BA et al . Comparison of endourological and open cystolithotomy in the management of bladder stones in children. J Urol. 2009 ;181:2684-7.
11. Ener K, Agras K, Aldemir M, et al. The randomized comparison of two different endoscopic techniques in the management of large bladder stones :transurethral use of nephroscope or cystoscope ? J Endourol. 2009; $23: 1151-5$. 